\title{
Brain Imaging in Patients with Transient Ischemic Attack: A Comparison of Computed Tomography and Magnetic Resonance Imaging
}

\author{
A. Förster ${ }^{\mathrm{a}} \quad$ A. Gass ${ }^{\mathrm{a}} \quad$ R. Kern ${ }^{\mathrm{a}} \quad$ H. Ay ${ }^{\mathrm{b}} \quad$ A. Chatzikonstantinou ${ }^{\mathrm{a}} \quad$ M.G. Hennerici ${ }^{\mathrm{a}}$ \\ K. Szabo ${ }^{a}$ \\ a Department of Neurology, Universitätsklinikum Mannheim, University of Heidelberg, Mannheim, Germany; \\ ${ }^{b}$ Departments of Neurology and Radiology, Massachusetts General Hospital, Harvard Medical School, \\ Boston, Mass., USA
}

Key Words

Transient ischemic attack $\cdot$ TIA $\cdot C T \cdot M R I$

\begin{abstract}
Background: Brain imaging in stroke aims at the detection of the relevant ischemic tissue pathology. Cranial computed tomography $(\mathrm{CT})$ is frequently used in patients with transient ischemic attack (TIA) but no data is available on how it directly compares to magnetic resonance imaging (MRI). Methods: We compared detection of acute ischemic lesions on CT and MRI in 215 consecutive TIA patients who underwent brain imaging with either CT $(n=161)$ or MRI $(n=54)$. An MRI was performed within $24 \mathrm{~h}$ in all patients who had CT initially. Results: An initial assessment with CT revealed no acute pathology in 154 (95.7\%) and possible acute infarction in $7(4.3 \%)$ patients. The acute infarct on CT was confirmed by diffusion-weighted imaging (DWI) in only 2 cases ( $28.6 \%$ ). DWI detected an acute infarct in 50 of the 154 patients with normal baseline CT (32.5\%). Among 54 patients without baseline CT, DWI showed acute ischemic lesions in 19 (35.2\%). The ischemic lesions had a median volume of $0.87 \mathrm{~cm}^{3}$ (range: $0.08-15.61$ ), and the lesion pattern provided clues to
\end{abstract}

the underlying etiology in $13.7 \%$. Conclusion: Acute MRI is advantageous over CT to confirm the probable ischemic nature and to identify the etiology in TIA patients.

Copyright ๑ 2012 S. Karger AG, Basel

\section{Introduction}

The term transient ischemic attack (TIA) was first introduced in 1964 [1] to describe a transient focal neurological deficit with restitution within $24 \mathrm{~h}$, a most likely cerebrovascular etiology and a presumed lack of permanent brain injury. The developments in brain imaging with the introduction of computed tomography (CT) and later magnetic resonance imaging (MRI) resulted in a changed point of view, a debate about this classical concept and a call for its replacement or redefinition [2]. In contrast to the assumption that neurological symptoms lasting less than $24 \mathrm{~h}$ are not associated with cerebral ischemia, several studies could demonstrate that up to $20 \%$ of TIA patients had a cerebral infarction on CT performed days to months after the episode $[3,4]$. The rate of cerebral infarctions is even higher - up to $67 \%$ - in TIA

\section{KARGER}

Fax +41613061234

E-Mail karger@karger.ch

www.karger.com
(C) 2012 S. Karger AG, Basel

0014-3022/12/0673-0136\$38.00/0

Accessible online at:

www.karger.com/ene
Alex Förster, MD

Department of Neurology, Universitätsmedizin Mannheim

Theodor-Kutzer-Ufer 1-3

DE-68167 Mannheim (Germany)

Tel. +49621383 2885, E-Mail foerster@ neuro.ma.uni-heidelberg.de 
patients who underwent MRI with diffusion-weighted imaging (DWI) $[5,6]$. While CT may be useful in excluding other etiologies of short-lasting focal neurological symptoms such as brain tumors or intracranial hemorrhage, its low sensitivity in detecting acute and small cortical and subcortical infarctions is a concern [7]. Only recently, Totaro et al. [8] could demonstrate that DWI is able to detect acute ischemic lesions in up to $58 \%$ of ischemic stroke patients in whom CT did not yield a definitive diagnosis in the subacute phase. There is currently no data on direct comparative assessments of CT and MRI in TIA patients in the acute situation. The objective of this study was to compare CT and MRI findings of acute cerebral infarction in patients with TIA.

\section{Patients and Methods}

For this retrospective observational study, we identified 215 consecutive patients with TIA from our Stroke Unit registry. Patients were eligible for the present study if (1) they had clinically suspected TIA with complete resolution of symptoms within $24 \mathrm{~h}$ after onset and before admission to our Stroke Center and (2) underwent either MRI or CT at admission and a second MRI within $24 \mathrm{~h}$ of the initial imaging. The decision whether a CT or MRI was performed as first-line imaging procedure was mainly based on the discretion of the treating physician and the availability of an MRI. All clinical and imaging data were recorded and documented in the framework of a standardized acute stroke care protocol: detailed physical and neurological examinations (4 times a day), assessment of cerebrovascular risk factors, CT and MRI, Doppler and duplex sonography of the extracranial vessels, transcranial Doppler sonography, transthoracic or transesophageal echocardiography, 24-hour Holter electrocardiogram and blood pressure monitoring, pulse oximetry and laboratory tests.

CT was performed with a Siemens Volume Zoom CT scanner (Siemens Medical Systems, Erlangen, Germany) with a slice thickness set to 4 and $5 \mathrm{~mm}$ for the posterior fossa and supratentorial scans, respectively ( $120 \mathrm{kV}, 350$ or $400 \mathrm{mAs})$. MRI was performed on a 1.5-T MR system (Magnetom Sonata; Siemens Medical Systems). A standardized protocol was used in all patients: (1) transverse, coronal and sagittal localizing sequences followed by transverse oblique contiguous images aligned with the inferior borders of the corpus callosum (applied on sequences 2-6); (2) $\mathrm{T}_{2}$-weighted images; (3) $\mathrm{T}_{1}$-weighted images; (4) diffusion-weighted images; (5) $\mathrm{T}_{2}$-weighted fluid-attenuated inversion recovery images; (6) $\mathrm{T}_{2}{ }^{*}$-weighted images; (7) 3-dimensional time-of-flight MR angiography sequences of the circle of Willis and the neck arteries in coronal direction.

Lesions on MRI were further analyzed: localization of acute ischemic lesions on DWI was classified in anterior, middle and posterior cerebral artery territory, border zone, brainstem and cerebellum. Acute ischemic lesion size was measured on DWI by manually delineated regions of interest (ROI), summation of these areas in square centimeters on each section, and multiplication by the interslice spacing to determine the volume in cubic
Table 1. Definition of ischemic lesion patterns in TIA patients

\begin{tabular}{lll}
\hline Pattern & Description & Example (DWI) \\
\hline 1 & $\begin{array}{l}\text { Solitary ischemic } \\
\text { lesion }\end{array}$ & \\
& &
\end{tabular}

\begin{tabular}{ll}
\hline 2 & Multiple ischemic \\
lesions scattered in one \\
vascular territory
\end{tabular}
vascular territory

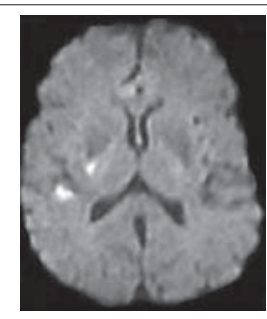

\begin{tabular}{ll}
\hline 3 & $\begin{array}{l}\text { Multiple ischemic } \\
\text { lesions scattered in } \\
\text { different vascular } \\
\text { territories, either in both } \\
\text { hemispheres or in the } \\
\text { anterior and posterior } \\
\text { circulation }\end{array}$ \\
\hline $4 \quad$ \\
Multiple ischemic \\
lesions scattered in one \\
vascular territory and \\
adjacent border zones
\end{tabular}

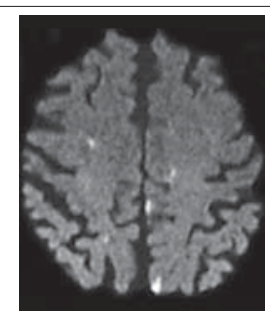
adjacent border zones

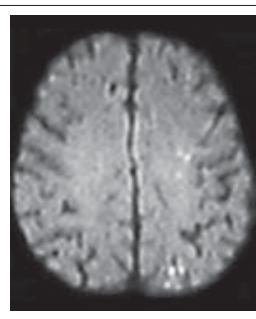

$5 \quad \begin{aligned} & \text { Solitary ischemic lesion } \\ & \text { in the territory of a deep } \\ & \text { penetrating artery }\end{aligned}$


Table 2. Baseline data of TIA patients

\begin{tabular}{|c|c|c|c|c|}
\hline & $\begin{array}{l}\text { All } \\
(n=215)\end{array}$ & $\begin{array}{l}\text { Initial CT } \\
(\mathrm{n}=161)\end{array}$ & $\begin{array}{l}\text { Initial MRI } \\
(\mathrm{n}=54)\end{array}$ & $\begin{array}{l}\mathrm{p} \\
\text { value }\end{array}$ \\
\hline Mean age $\pm S D$, years & $67.1 \pm 12.9$ & $68.1 \pm 13.0$ & $64.0 \pm 12.0$ & 0.04 \\
\hline Male sex, n (\%) & $116(54.0)$ & $86(53.4)$ & $30(55.6)$ & 0.79 \\
\hline \multicolumn{5}{|l|}{ Time of onset to admission } \\
\hline Mean exact time $\pm S D, \min (n=112)$ & $195.5 \pm 144.71$ & $188.9 \pm 139.0$ & $213.9 \pm 160.7$ & 0.40 \\
\hline \multicolumn{5}{|l|}{ Duration of symptoms } \\
\hline \multirow{2}{*}{\multicolumn{5}{|c|}{ 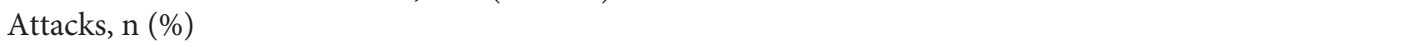 }} \\
\hline & & & & \\
\hline \multicolumn{2}{|l|}{ Medical history, n (\%) } & $125(77.6)$ & $38(70.4)$ & 0.28 \\
\hline $\begin{array}{l}\text { Hedical nistory, } \mathrm{n}(\%) \\
\text { Hypertension }\end{array}$ & $167(77.7)$ & $127(78.9)$ & $40(74.1)$ & 0.46 \\
\hline Diabetes mellitus & $40(18.6)$ & $26(16.1)$ & $14(25.9)$ & 0.11 \\
\hline Hyperlipidemia & $81(37.7)$ & $58(36.0)$ & $23(42.6)$ & 0.39 \\
\hline History of smoking & $29(13.5)$ & $23(14.3)$ & $6(11.1)$ & 0.56 \\
\hline Coronary heart disease & $39(18.1)$ & $29(18.0)$ & $10(18.5)$ & 0.93 \\
\hline Peripheral vascular disease & $15(7.0)$ & $11(6.8)$ & $4(7.4)$ & 0.89 \\
\hline Prior TIA & $16(7.4)$ & $13(8.1)$ & $3(5.6)$ & 0.54 \\
\hline Prior stroke & $21(9.8)$ & $17(10.6)$ & $4(7.4)$ & 0.50 \\
\hline \multicolumn{5}{|l|}{ Treatment with, n (\%) } \\
\hline Platelet inhibitors & $71(33.0)$ & $52(32.3)$ & $19(35.2)$ & 0.70 \\
\hline Therapy with anticoagulants & $14(6.5)$ & $13(8.1)$ & $1(1.9)$ & 0.11 \\
\hline
\end{tabular}

centimeters using a commercially available workstation (nordicICE, NordicNeuroLab, Bergen, Norway). Both CT and MRI were assessed by an attending neuroradiologist/neurologist for clinical purposes and by a study physician (A.F.) who was provided with the clinical information.

Based on the lesion distribution and localization on DWI, 5 patterns of ischemic lesions were defined (summarized in table 1). Pattern 1 is defined as a solitary ischemic lesion involving the cerebral cortex and/or subcortical structures. Pattern 2 represents multiple ischemic lesions either in cortical or subcortical regions in one vascular territory. Pattern 3 encompasses several disseminated small lesions sprinkled in different vascular territories either in both hemispheres or in the anterior and posterior circulation, involving mainly but not exclusively cortical regions. This ischemic lesion pattern is considered to be typical for stroke due to a proximal embolic source (the heart or proximal vessels). Pattern 4 denotes multiple ischemic lesions either completely or predominantly located in regions considered to be one of the hemodynamic risk zones between major cerebrovascular territories: the superficial or cortical border zones wedged between the anterior and middle cerebral artery or between the middle and posterior cerebral artery, and the deep or subcortical border zone located in the vascular territory between deep and superficial arterial systems. This ischemic lesion pattern is assumed to be characteristic for ischemic stroke due to large artery disease. Pattern 5 is defined as infarction in the territory of a deep perforating artery originating from the middle cerebral artery, posterior cerebral artery or basilar artery.

Probable TIA/stroke etiology was classified according to the Trial of Org 10172 in Acute Stroke Treatment (TOAST) classifica- tion [9] as large vessel disease, small vessel occlusion, cardioembolism, other rare causes, and undetermined causes as implemented earlier [10].

Statistical analysis was carried out using SPSS 19.0. Descriptive data was analyzed using either t tests, the rank-sum test or $\chi^{2}$-based tests as appropriate.

\section{Results}

Between July 2007 and September 2008, 215 consecutive patients (mean age: 67.1 years, range: $31-92$ ) were included in the study. Of these, 116 (54.0\%) were men, and $99(46.0 \%)$ were women. Table 2 shows the baseline characteristics. Based on the clinical presentation, TIA was classified as localized in the anterior circulation in 111 (51.6\%) patients, in the posterior circulation in only 38 patients (17.7\%); localization was classified as uncertain in 66 patients $(30.7 \%)$. Exact onset of symptoms could be identified in only 145 (67.4\%), while the exact duration of symptoms (mean duration: $40.4 \mathrm{~min}$ ) could be estimated in 112 (52.1\%) patients. In the remaining 103 patients, the duration of symptoms could be narrowed down to minutes in $52(50.5 \%)$ or hours in $51(49.5 \%)$ patients. In all patients, clinical symptoms resolved completely before admission. 
Fig. 1. CT and MRI findings in a 49-yearold TIA patient. CT (a), diffusion-weighted (b) and $\mathrm{T}_{2}$-weighted FLAIR MRI (c) of a 49-year-old man presenting with transient nausea, dizziness and right visual field loss. CT, diffusion-weighted and $\mathrm{T}_{2^{-}}$ weighted FLAIR images show a small acute ischemic lesion (arrow) in the left posterior cerebral artery territory.
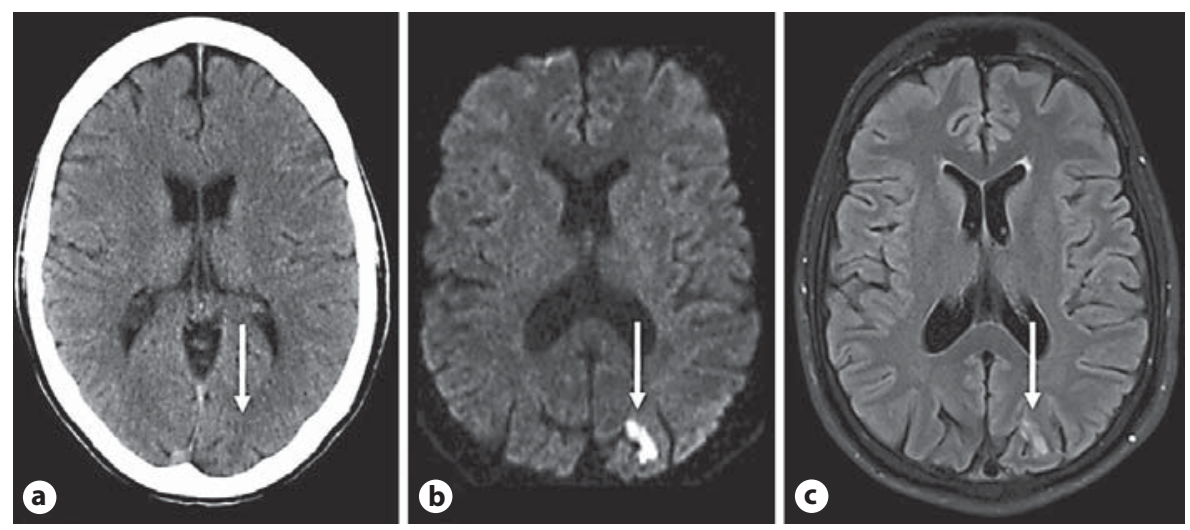

All patients underwent early brain imaging, 161 of 215 (74.9\%) had both CT and MRI, while 54 of 215 (25.1\%) had MRI only. There was no difference with regard to baseline characteristics (see table 2), clinical presentation, and etiology between patients with and without CT except for a higher age in the CT group. A difference in image interpretation between the attending neuroradiologist/neurologist and the study physician was identified in only one case (a CT scan was read as normal by the study physician, while a lesion was seen by the attending neuroradiologist). Assessment of CT images revealed no acute pathology in 154 (95.7\%) and possible acute infarction in 7 (4.3\%) patients; of these suspected lesions, 6 were located in the anterior circulation and 1 in the posterior circulation. DWI confirmed the acute infarct on CT in only 2 of the 7 patients (28.6\%); 1 in the anterior circulation and 1 in the posterior circulation, respectively (see fig. 1). DWI detected an acute infarct in 50 patients with an initially normal CT (32.5\%). Among the 54 TIA patients who did not have baseline CT examination, DWI showed acute ischemic lesions in 19 (35.2\%). In none of the remaining patients did follow-up MRI detect new ischemic lesions. DWI demonstrated a median number of acute ischemic lesions of 1 (range: 1-14). Most patients had lesions in the superficial (71.2\%) and deep (47.9\%) middle cerebral artery territory followed by the posterior cerebral artery territory $(6.8 \%)$ and the cerebellum (5.5\%). The most frequent ischemic lesion patterns were pattern $1(45.2 \%)$ and pattern 2 (37.0\%), followed by pattern $3(6.8 \%), 4(4.1 \%)$, and $5(6.8 \%)$. The latter 3 provided clues for the underlying etiology in 13.7\% of cases: pattern 3 and diagnosis of cardioembolism $(\mathrm{n}=3)$, pattern 4 and diagnosis of large vessel disease $(\mathrm{n}=$ 3), and pattern 5 and final diagnosis of small vessel occlusion $(\mathrm{n}=4)$. The ischemic lesions had a median volume of $0.87 \mathrm{~cm}^{3}$ (range: $0.08-15.61$ ). Patients with suspected TIA
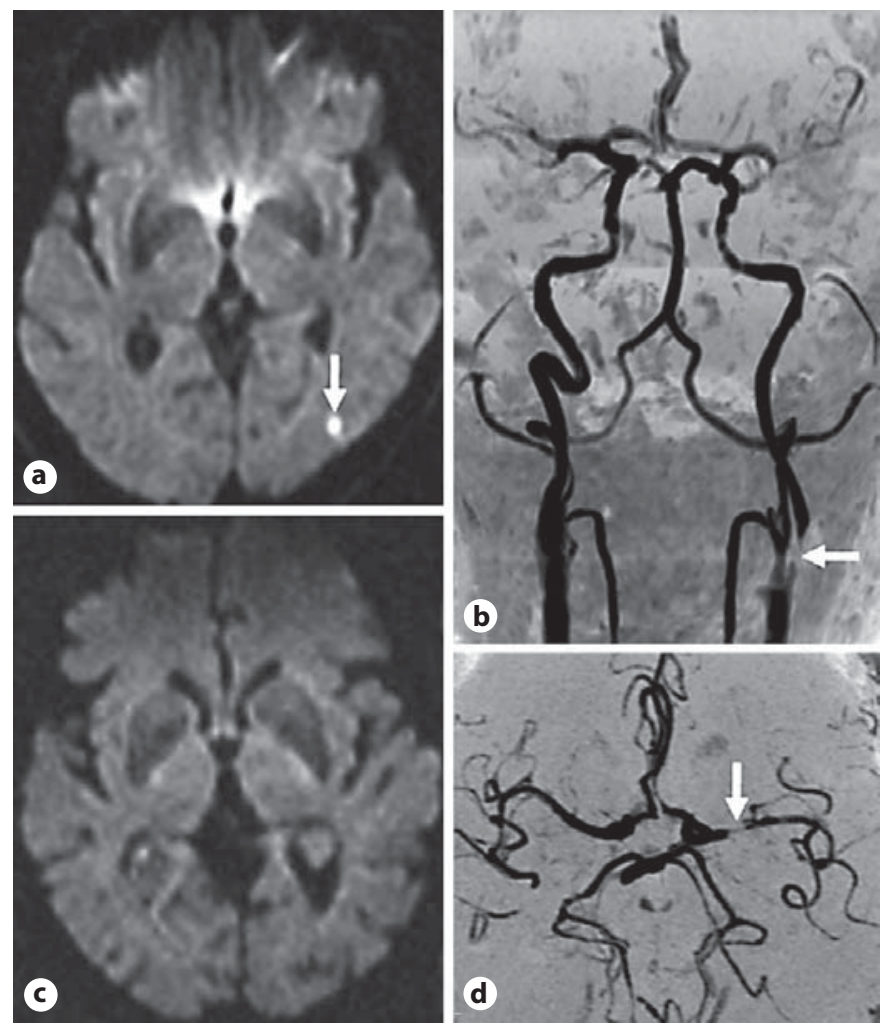

Fig. 2. DWI and MR angiography findings in two patients with TIA. In the first patient, DWI shows an acute ischemic lesion (arrow) in the left middle cerebral artery territory (a). MR angiography demonstrates a proximal left internal carotid artery stenosis (b). In the second patient, DWI is unremarkable (c); MR angiography shows a stenosis of the left middle cerebral artery (d).

in the anterior circulation (59.5\%) had significantly higher rates of acute ischemic lesions on DWI compared to those with suspected TIA in the posterior circulation $(13.2 \%)$ and with uncertain localization $(3.0 \%$; $p<0.001)$. 
With regard to gender, duration and number of the attacks, common risk factors, and treatment with platelet inhibitors or anticoagulants, there were no differences between patients with suspected TIA in the anterior and posterior circulation. MR angiography detected a stenosis or occlusion of the extracranial or intracranial vessels in $12(5.6 \%)$ patients. In detail, there was a stenosis or occlusion of the proximal or distal internal carotid artery in 10 (4.7\%) patients and of the middle cerebral artery in 2 (0.9\%) patients (see fig. 2 ).

Etiologic stroke subtypes remained undetermined in $60.9 \%$ of all TIA patients. In contrast to this, there was a significantly lower rate of undetermined causes in patients with acute DWI lesion ( 45.2 vs. $69.0 \%$, OR $0.37,95 \%$ CI $0.21-0.66 ; p=0.001$ ), and cardiogenic causes of embolism were identified more often in patients with positive DWI (35.6 vs. $14.8 \%$, OR 3.19, 95\% CI 1.64-6.29; p < 0.001).

\section{Discussion}

In this single-center study, we demonstrate that CT, as the initial line of imaging, rarely provides positive findings with regard to the suspected ischemic lesion, as the clinically relevant acute infarct was captured by CT in just $4.3 \%$ of patients. Previous publications have reported detection rates of subacute ischemic lesions on CT in up to $20 \%$ of patients after several days to months $[3,4]$ underlining its low sensitivity in the acute work-up of TIA patients. In contrast to CT, DWI showed an acute infarct in approximately one third of the patients. The overall agreement rate between DWI and CT was $68.7 \%$, meaning that, approximately in one third of TIA patients, CT either misses or misidentifies the ischemic lesion on DWI. These finding have major implications with regards to the clinical applicability of the recently proposed tissue-based definition of TIA. Although the new AHA definition explicitly recognizes MRI as the first choice of imaging in TIA, it still reserves room for CT as an alternative option [11]. Here, we quantify the difference be- tween these two options: $31.3 \%$ of patients are misclassified by the tissue-based definition if CT is used instead of DWI. The remarkable difference between CT and MRI may justify a more sound recommendation in favor of MRI for a consistent classification by the tissue-based definition. The major advantage of MRI over CT is its ability to demonstrate very small acute ischemic lesions on DWI [12], which has a very short acquisition time of about $60 \mathrm{~s}$. As demonstrated in this work, DWI not only confirms the clinical diagnosis, but also facilitates the recognition of certain patterns of ischemia, thereby providing clues to the underlying etiology. Finally, the presence of acute lesions can also improve the prediction of stroke risk after TIA [13].

The present study has some limitations. First, CT and MRI were not obtained right after one another and some of the lesions missed by CT might have occurred in the interim. Second, some DWI lesions might have been missed due to early reversal in the interim. However, both scenarios are unlikely to have significantly affected the results as the rate of proven ischemic lesions on DWI was similar in patients who underwent MRI only to those who had CT first and then MRI.

Thus, the use of MRI for the management of TIA is advantageous for diagnosis because of its ability to detect hyperacute and minute ischemic lesions as well as ischemic lesions in the posterior fossa. Therefore, it seems important to perform a thorough MRI examination in all patients with clinically suspected TIA. MRI, on the other hand, is expensive, not widely available, and limited by contraindications. Nevertheless, our findings suggest that, as proposed by the most recent TIA guidelines, MRI should be the preferred line of imaging in all patients with suspected TIA whenever it is available and feasible.

\section{Acknowledgements}

The authors have no conflict of interest related to the present study. Dr. Ay has been supported by NIH grant R01-NS059710.

\section{References}

1 Marshall J: The natural history of transient ischaemic cerebro-vascular attacks. Q J Med 1964:33:309-324.

-2 Albers GW, Caplan LR, Easton JD, Fayad PB, Mohr JP, Saver JL, Sherman DG: Transient ischemic attack - proposal for a new definition. N Engl J Med 2002;347:1713-1716.

\footnotetext{
-3 Evans GW, Howard G, Murros KE, Rose LA, Toole JF: Cerebral infarction verified by cranial computed tomography and prognosis for survival following transient ischemic attack. Stroke 1991;22:431-436.

$\checkmark 4$ Davalos A, Matias-Guiu J, Torrent O, Vilaseca J, Codina A: Computed tomography in reversible ischaemic attacks: clinical and prognostic correlations in a prospective study. J Neurol 1988;235:155-158.
} 
5 Ay H, Oliveira-Filho J, Buonanno FS, Schaefer PW, Furie KL, Chang YC, Rordorf G, Schwamm LH, Gonzalez RG, Koroshetz WJ: 'Footprints' of transient ischemic attacks: a diffusion-weighted MRI study. Cerebrovasc Dis 2002;14:177-186.

-6 Rovira A, Rovira-Gols A, Pedraza S, Grive E, Molina C, Alvarez-Sabin J: Diffusionweighted MR imaging in the acute phase of transient ischemic attacks. AJNR Am J Neuroradiol 2002;23:77-83.

$\checkmark 7$ Mullins ME, Schaefer PW, Sorensen AG, Halpern EF, Ay H, He J, Koroshetz WJ, Gonzalez RG: CT and conventional and diffusion-weighted MR imaging in acute stroke: study in 691 patients at presentation to the emergency department. Radiology 2002; 224:353-360.

8 Totaro P, Toni D, Durastanti L, Bozzao L, Gualdi GF, Raz E, Kouleridou A, Pantano P: Diffusion-weighted MRI in patients with non-diagnostic CT in the post-acute phase of cerebral ischemia. Eur Neurol 2010;63:94100.

$\checkmark 9$ Adams HP, Bendixen BH, Kappelle LJ, Biller J, Love BB, Gordon DL, Marsh EE, and the TOAST Investigators: Classification of subtype of acute ischemic stroke. Definitions for use in a multicentre clinical trial. Stroke 1993;24:35-41.

10 Purroy F, Montaner J, Rovira A, Delgado P, Quintana M, Alvarez-Sabin J: Higher risk of further vascular events among transient ischemic attack patients with diffusionweighted imaging acute ischemic lesions. Stroke 2004;35:2313-2319.

11 Easton JD, Saver JL, Albers GW, Alberts MJ, Chaturvedi S, Feldmann E, Hatsukami TS, Higashida RT, Johnston SC, Kidwell CS, Lutsep HL, Miller E, Sacco RL: Definition and evaluation of transient ischemic attack: a scientific statement for healthcare profession- als from the American Heart Association/ American Stroke Association Stroke Council; Council on Cardiovascular Surgery and Anesthesia; Council on Cardiovascular Radiology and Intervention; Council on Cardiovascular Nursing; and the Interdisciplinary Council on Peripheral Vascular Disease. The American Academy of Neurology affirms the value of this statement as an educational tool for neurologists. Stroke 2009;40: 2276-2293.

12 Gass A, Ay H, Szabo K, Koroshetz WJ: Diffusion-weighted MRI for the 'small stuff': the details of acute cerebral ischaemia. Lancet Neurol 2004;3:39-45.

13 Ay H, Arsava EM, Johnston SC, Vangel M, Schwamm LH, Furie KL, Koroshetz WJ, Sorensen AG: Clinical- and imaging-based prediction of stroke risk after transient ischemic attack: the CIP model. Stroke 2009;40:181186. 